\title{
RATE-ADAPTIVE CODES FOR THE ENTIRE SLEPIAN-WOLF REGION AND ARBITRARILY CORRELATED SOURCES
}

\author{
V. Toto-Zarasoa, A. Roumy and C. Guillemot \\ INRIA/IRISA, Campus Universitaire de Beaulieu, 35042 Rennes-Cedex, France.
}

\begin{abstract}
In this paper, we focus on the design of distributed source codes that can achieve any point in the Slepian-Wolf (SW) region and at the same time adapt to any correlation between the sources. A practical solution based on punctured accumulated LDPC codes extended to the non asymmetric case is described. The approach allows flexible rate allocation to the two sources with a gap of 0.0677 bits with respect to the minimum achievable rate.
\end{abstract}

Index Terms - Data compression, source coding, channel coding, error correction coding, block codes.

\section{INTRODUCTION}

Distributed source coding (DSC) refers to the problem of compressing correlated sources. DSC finds its foundation in the seminal Slepian-Wolf [1] and Wyner-Ziv [2] theorems. In the sequel, we consider only the Slepian-Wolf lossless coding problem of two correlated binary sources $X$ and $Y$. If there is cooperation between the coders of $X$ and $Y$, the problem reduces to the single user Shannon's source coding problem and the rates $R_{X}$ and $R_{Y}$ achievable for the two sources verify the sum rate condition $R_{X}+R_{Y} \geq H(X, Y)$. If there is no cooperation between the two coders, Slepian and Wolf (SW) have shown [1] that the sum rate condition remains $R_{X}+R_{Y} \geq H(X, Y)$ with the constraints $R_{X} \geq H(X \mid Y)$, $R_{Y} \geq H(Y \mid X)$, (see Fig. 1) provided that the two sources are decoded jointly.

DSC has gained increasing interest recently for a range of applications such as wireless sensor networks (WSN), video compression, loss resilient video transmission, or analog television upgrade using a digital side channel. First attempts to construct DSC codes have considered the so-called asymmetric DSC problem, or the problem of coding with side information, corresponding to the corner points ( $A$ and $B$ in Fig. 1) of the $\mathrm{SW}$ rate region. These constructions are motivated by the optimality of capacity-achieving channel codes for the asymmetric DSC problem [2]. The statistical dependence between the two sources is modeled as a virtual correlation channel

This research was partly funded by the French National Research Agency (ANR) under the Essor project and by the European Commission IST FP6 program under the DISCOVER project (http://www.discoverdvc.org). analogous to a binary symmetric channel (BSC) defined by a cross-over probability $p=\mathbb{P}(X \neq Y)$. The source $Y$ (called the side information) is thus regarded as a noisy version of $X$ (called the main signal). The side information $Y$ is transmitted at full rate (i.e. $R_{Y}=H(Y)$ ), whereas the other source is compressed either by sending a syndrome or parity bits. The first practical asymmetric Slepian-Wolf coding solution called DIstributed Source Coding Using Syndromes (DISCUS) has been proposed in [3]. The transmission of a syndrome (or coset index) instead of the complete codeword yields compression of the information. The receiver decodes the value of $X$ by choosing the codeword in the given coset that is closest in terms of Hamming distance to the binary representation of the symbols of the source that serves as side information $Y$. To approach the Slepian-Wolf bounds more closely, efficient codes such as Turbo Codes [4][5][6][7] and LDPC codes [8][9] have also been considered.

Code design has then been recently extended to the case where both sources are compressed, in order to reach any point of the segment between $A$ and $B$, for a given crossover probability $p$. We refer to this set-up as non asymmetric DSC and to symmetric DSC, when both sources are compressed at the same rate. Both syndrome-based approaches (symmetric DSC [10][11], and [12][13] for non asymmetric) and parity-based approaches (symmetric [4], non asymmetric [14][7]) have been considered. Syndrome-based approaches are known to be optimal, however maybe less amenable to rate adaptation. Yet, rate-adaptive symmetric codes may be beneficial for applications such as multi-view light field coding [11] or for joint optimization of the rate and power of transmission in a sensor network application [15]. For example, in a WSN, spatially distributed sensors gather data and send them to a common center (or sink). It is shown in [15] that the optimal rate allocation depends on the transmission conditions and can therefore be any point in the SW region. It is therefore of interest to construct DSC codes that can achieve any point in the SW region. Moreover to meet the low cost constraint, a single sensor must be designed that can handle any correlation between the sources.

Here, we focus on the design of a single DSC code that achieves any point in the SW region but also adapts to any correlation between the sources, hence the name rate-adaptive non asymmetric codes. We consider a punctured syndrome- 
based approach based on accumulated LDPC codes [16] which are thus extended the non asymmetric case. Syndrome and systematic bits are transmitted for both sources in a respective amount depending on the rate allocated to the two sources. The adaptation of the rate to varying correlation is performed via a puncturing of the syndrome information.

\section{SLEPIAN-WOLF CODING}

\subsection{Theoretical results}

Let $X$ and $Y$ be two binary correlated memoryless sources. The minimum achievable rate for lossless compression of the two sources $X$ and $Y$ is given by the joint entropy $H(X, Y)$. To approach this rate bound, practical systems encode and decode the two sources jointly. However, Slepian and Wolf have established in 1973 [1] that this lossless compression rate bound could also be approached with a vanishing error probability for long sequences when encoding the two sources separately and decoding them jointly, as depicted in Fig. 1. The achievable rate region established by the Slepian-Wolf theorem is thus given by $R_{X} \geq H(X \mid Y), R_{Y} \geq H(Y \mid X)$, and $R_{X}+R_{Y} \geq H(X, Y)$. The corner points $A$ and $B$ of the

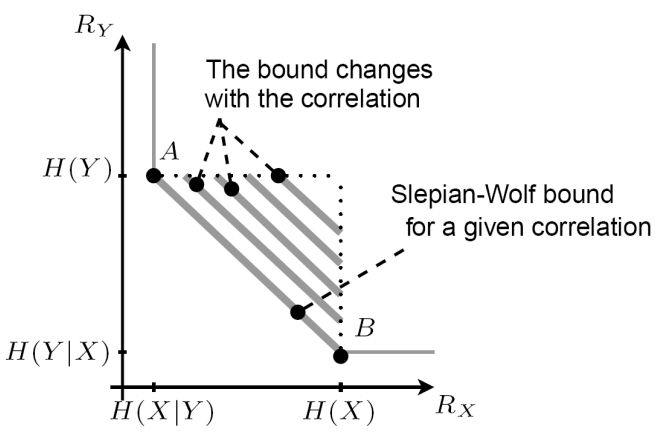

Fig. 1. Slepian-Wolf rate region.

Slepian-Wolf rate region correspond to the asymmetric setup, whereas all the points between $A$ and $B$ correspond to the non asymmetric DSC case. When the correlation decreases, the rate bound moves away from the origin (see Fig. 1).

\subsection{Syndrome-based coding}

The use of parity-check codes for approaching the corner points of the Slepian-Wolf rate region was first suggested in 1974 [2]. It is suggested to construct bins as cosets of a capacityachieving parity-check code. A $(n, k)$ code partitions the space of $2^{n}$ sequences into $2^{n-k}$ "cosets" containing $2^{k}$ words with maximum Hamming distance. Each coset is indexed by an $(n-k)$-length syndrome. Let $x$ and $y$ be two correlated binary sequences of length $n$. These sequences are the realizations of the sources $X$ and $Y$. The coder computes and transmits the syndrome of $x, s_{x}$. The sequence $x$ of $n$ input bits is thus mapped into its corresponding $(n-k)$ syndrome bits, leading to a compression ratio of $n:(n-k)$. The decoder, knowing the correlation between the sources $X$ and $Y$ and the coset index $s_{x}$, searches for the sequence in the coset that is closest to $y$. In other words, maximum likelihood decoding ${ }^{1}$ is performed in order to retrieve the original $x$ sequence. Considering LDPC codes, the search for the closest sequence is done with an approximated maximum likelihood decoder: the sum-product algorithm.

The syndrome-based approach is optimum in the sense of approaching the Slepian-Wolf bound. However, rate adaptation, e.g., using puncturing mechanisms is not natural. When syndrome bits are punctured, the decoder should in principle search for the closest sequence in a union of cosets, which would require modifying the sum-product decoder. If the sum-product decoder is not modified, then the syndrome bits (of degree one in the LDPC graph) are not protected, which leads to systematic errors. A brute force decoder consists in performing a sum product algorithm, but then the decoding complexity of such a method would grow exponentially with the number of punctured bits. The authors in [16] suggested accumulating the syndrome bits before the puncturing step, thus maintaining the performance of the code. The proposed approach however is appropriate for the asymmetric set-up only. In the sequel, the approach is extended in order to cover the entire Slepian-Wolf rate region, that is not limited to the asymmetric set-up.

\section{CODING FOR THE ENTIRE SLEPIAN-WOLF REGION}

\subsection{Non asymmetric SW coding for a given correlation}

Let $X$ and $Y$ be two random binary correlated sources of a given correlation defined by the cross-over probability $p$. Let $x$ and $y$ be their realizations of length $n$. Let us consider an $(n, k)$ LDPC code defined by its parity-check matrix $H_{(n-k) \times n}=\left[A_{(n-k) \times k} B_{(n-k) \times(n-k)}\right]$. The syndromes $s_{x}=H . x$ and $s_{y}=H . y$, of length $(n-k)$, are computed for both sequences and transmitted to the decoder. In addition, the $k^{\prime}$ first bits of $x\left(x_{1}^{k^{\prime}}\right)$ and the $k-k^{\prime}$ next bits for the source $y\left(y_{k^{\prime}+1}^{k}\right)$, are transmitted as systematic bits, where $k^{\prime}$ is an integer so that $k^{\prime} \in[0, k]$. The total rate for the sources $X$ and $Y$ is respectively $n-k+k^{\prime}$ and $n-k^{\prime}$ bits. The structure of the coders is depicted in Fig. 2. Note that $k^{\prime}=k$ and $k^{\prime}=0$ correspond to the two asymmetric set-ups with rates given by the corner points $A$ and $B$ of the SW region.

The decoder computes first $s_{z}=s_{x} \oplus s_{y}$, which turns out to be the syndrome of the error pattern $z$ between $x$ and $y$. A modified LDPC decoder estimates $\hat{z}=\hat{x} \oplus \hat{y}$ from the syndrome $s_{z}$ and the all-zero word of size $n$ (see Fig. 3) [8]. More precisely, $z$ corresponds to the smallest weight vector

\footnotetext{
${ }^{1}$ Note also, that the maximization is performed in a set of codewords with syndrome that may not be 0 .
} 


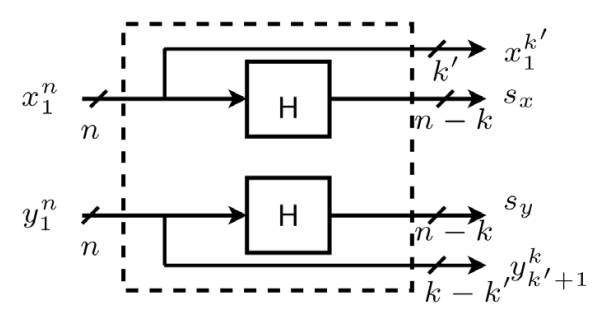

Fig. 2. The symmetric coders.

with syndrome $s_{z}$, or in other words, $z$ is the vector closest to the all-zero word among the vectors with syndrome $s_{z}$.

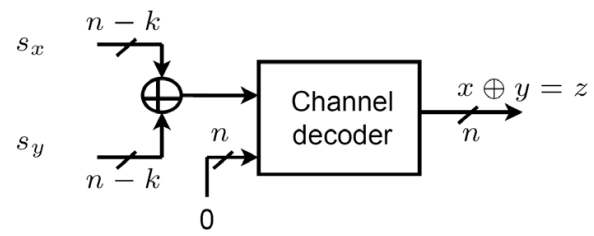

Fig. 3. First step in the decoding process: estimation of the error pattern $z=x \oplus y$.

Once the difference pattern is found, $x_{k^{\prime}+1}^{k}$ and $y_{1}^{k^{\prime}}$ can be estimated thanks to the relation $z=x \oplus y: x_{k^{\prime}+1}^{k}=y_{k^{\prime}+1}^{k} \oplus$ $z_{k^{\prime}+1}^{k}$ and $y_{1}^{k^{\prime}}=x_{1}^{k^{\prime}} \oplus z_{1}^{k^{\prime}}$. The subsequences $x_{k+1}^{n}$ and $y_{k+1}^{n}$ then remain to be computed. Since, $s_{x}=[A B] x=A x_{1}^{k} \oplus$ $B x_{k+1}^{n}$, then $x_{k+1}^{n}=B^{-1}\left[s_{x} \oplus A x_{1}^{k}\right]$, where $B^{-1}$ denotes the inverse of the matrix $B$ [11][13]. At this stage, we can see why a full-rank matrix $B$ is mandatory for this algorithm to work. Note that for a rate $k / n$ channel code, the parity check matrix $H$ has rank $n-k$ which insures that $n-k$ columns of $H$ are free; then a permutation matrix, say $P_{n \times n}$, is found so that the matrix $H$ has the right form.

\subsection{Non asymmetric SW coding for varying correlation}

We now consider the problem of adapting the rate of the above coding/decoding system to varying correlation, by puncturing some syndrome bits. To avoid degrading the performance of the LDPC code, the syndrome bits are first accumulated before being punctured as suggested in [16]. This accumulating allows to protect the punctured syndrome bits, with a code of rate 1 , so that the compression rate is not modified. The effect of this accumulator code is equivalent to merging some rows of the parity check matrix by adding them.

A set of $N$ matrices $\left(H_{i}\right)_{i=1 \ldots N}$ of sizes $\left(n-k_{i}\right) \times n, i=$ $1 \ldots N$ corresponding to $N$ LDPC codes is considered. These matrices are built according to [16]. Without loss of generality, assume that $\forall i, j \in[1, N], i<j \Rightarrow\left(n-k_{i}\right)<\left(n-k_{j}\right)$, meaning that the $N$ matrices have a growing number of rows. Also consider the set of permutation matrices $\left(P_{i}\right)_{i=1 \ldots N}$ of size $n \times n$ so that $\forall i \in[1, N], H_{i} P_{i}$ has an invertible $B$

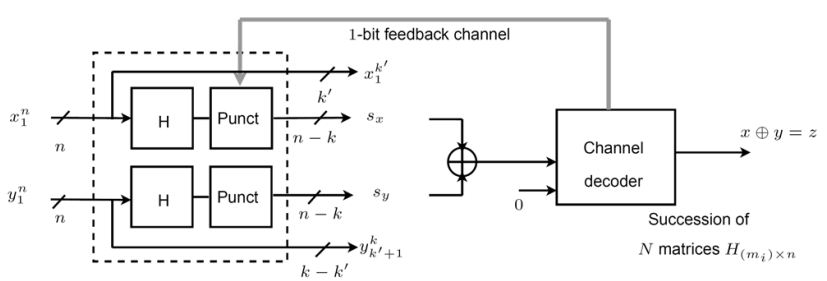

Fig. 4. The single rate-adaptive codec.

part. These permutation matrices are not all the same because merging the rows of the original matrix $H$ moves the location of the free columns. The coding and decoding structures are depicted in Fig. 4.

The coders send a first set of accumulated syndromes bits. With that information, the decoder tries to find a first estimate of the error pattern $z$. If the iterative algorithm does not converge, that is $\hat{z}$ does not satisfy the condition $H \hat{z}=s_{z}$, more syndrome bits are requested to the encoder via a one-bit feedback channel, and this until $\hat{z}$ has syndrome $s_{z}$.

Let $H_{i}$ be the matrix of stage $i$ at which $z$ is found. Both the coder and the decoder know which $\left(n-k_{i}\right)$ columns of $H_{i}$ are free. Let $k^{\prime}$ be an integer so that $k^{\prime} \in\left[0, k_{i}\right]$. The systematic bits for both sources can then be sent. The source $X$ transmits the $k^{\prime}$ bits corresponding to the $k^{\prime}$ first columns of the $k_{i}$ columns of $H_{i}$ which are not free. $Y$ transmits the $k_{i}-k^{\prime}$ bits corresponding to the $k_{i}-k^{\prime}$ remaining non-free columns of $H_{i}$. Then $R_{X}=\frac{\left(n-k_{i}\right)+k^{\prime}}{n}$ and $R_{Y}=\frac{n-k^{\prime}}{n}$. In other words, the parameter $i$ first determines the global rate of the system, and $k^{\prime}$ fixes the specific rates of $X$ and $Y$. These rates can be optimized each time a new sequence of length $n$ is to be encoded.

\section{SIMULATIONS AND RESULTS}

The tests have been conducted with a set of matrices generated in the same way as in [16]. We consider input sequences of length $n=2046$ and generate 65 matrices having sizes ranging from $62 \times 2046$ to $2046 \times 2046$. The sources $X$ and $Y$ are i.i.d. random variables with a uniform binary distribution. The correlation between $X$ and $Y$ is modeled as a BSC with crossover probability $p$. In our tests, $p$ varies from 0.01 to 0.17 , with a step of 0.04 . For each correlation factor $p$, different compression rate allocations are tested in order to achieve any point of the SW boundary. This degree of freedom is obtained by tuning $k^{\prime}$ the number of input bits sent by source $X$. More precisely, 11 different values of $k^{\prime}$ are considered, varying from 0 to $k$, with a step of $0.1 * k$; for each $k^{\prime}, 500$ words are tested; that way, we test about $10^{7}$ bits for each $p$.

Our system has a bit error rate of $9 \times 10^{-5}$ and we are only 0.0677 bits away from the bound, on average, which is among the best results reported so far for a code of that size. 
The results and the corresponding theoretical bounds in the Slepian-Wolf region are reported in Fig. 5.

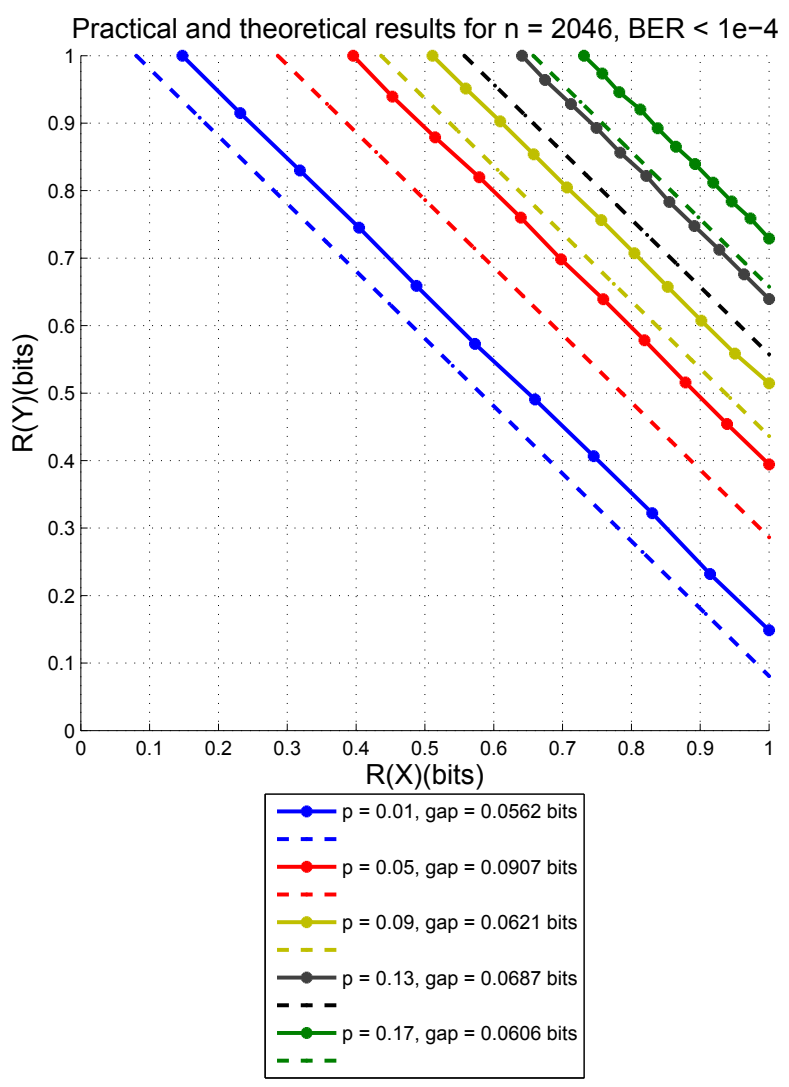

Fig. 5. Performance of the single rate adaptive codec. Achieved compression rates are plotted for different correlation parameters $p$ and compared with the optimal Slepian Wolf bounds.

\section{CONCLUSION}

We have designed a rate adaptive code for the non asymmetric lossless DSC problem. More precisely, a single code has been proposed that adapts to any variation in the correlation between the two sources and that also achieves any point in the Slepian Wolf region. The code is based on punctured accumulated codes. It is shown that the scheme needs a single codec. Moreover, the source statistics are not required at the encoder and the rate adaptation is performed with a feedback. Finally, the proposed scheme does not require to re-encode the data, while adapting the compression rate.

\section{REFERENCES}

[1] D. Slepian and J. K. Wolf, "Noiseless coding of correlated information sources," IEEE Transactions on Information Theory, vol. 19, no. 4, pp. 471-480, July 1973.
[2] A. Wyner, "Recent results in the shannon theory," IEEE Transactions on Information Theory, vol. 20, pp. 2-10, 1974.

[3] S. S. Pradhan and K. Ramchandran, "Distributed source coding using syndromes (discus): Design and construction," Proceedings of the IEEE Data Compression Conference, pp. 158-167, March 1999.

[4] J. Garcia-Frias and Y. Zhao, "Compression of correlated binary sources using turbo codes," IEEE Communications Letters, vol. 5, pp. 417-419, October 2001.

[5] J. Bajcsy and P. Mitran, "Coding for the slepian-wolf problem with turbo codes," in Proceedings Globecom, 2001, pp. 1400 1404.

[6] A. Aaron and B. Girod, "Compression with side information using turbo codes," in Proceedings of the IEEE Data Compression Conference, Apr 2002, pp. 252-261.

[7] J. Garcia-Frias and F. Cabarcas, "Approaching the slepian-wolf boundary using practical channel codes," Signal Processing, vol. 86, no. 11, pp. 3096-3101, 2006.

[8] A. D. Liveris, Z. Xiong, and C. N. Georghiades, "Compression of binary sources with side information at the decoder using ldpc codes," IEEE Communications Letters, vol. 6, no. 10, pp. 440-442, October 2002.

[9] T. Tian, J. Garcia-Frias, and W. Zhong, "Compression of correlated sources using ldpc codes," in Proceedings of the IEEE Data Compression Conference, Washington, DC, USA, 2003, p. 450, IEEE Computer Society.

[10] S. S. Pradhan and K. Ramchandran, "Distributed source coding: symmetric rates and applications to sensor networks," in Proceedings of the IEEE Data Compression Conference, March 2000, pp. 363-372.

[11] N. Gehrig and P. L. Dragotti, "Symmetric and asymmetric slepian-wolf codes with systematic and non-systematic linear codes," IEEE communications letters, vol. 9, no. 1, pp. 61-63, January 2005.

[12] V. Stankovic, A. D. Liveris, Z. Xiong, and C. N. Georghiades, "Design of slepian-wolf codes by channel code partitioning," in Proceedings of the IEEE Data Compression Conference, 2004, pp. 302-311.

[13] P. Tan and J. Li, "A practical and optimal symmetric slepianwolf compression strategy using syndrome formers and inverse syndrome formers," in Proceedings of 43rd Annual Allerton Conference on Communication, Control and Computing, September 2005.

[14] M. Sartipi and F. Fekri, "Distributed source coding in wireless sensor networks using ldpc coding: The entire slepian-wolf rate region," IEEE Wireless Communications and Networking Conference, vol. 4, pp. 1939-1944, March 2005.

[15] A. Roumy and D Gesbert, "Optimal matching in wireless sensor networks," IEEE Journal on Selected Topics in Signal Processing, Special issue on Convex Optimization Methods in Signal Processing, December 2007, in press.

[16] D. Varodayan, A. Aaron, and B. Girod, "Rate-adaptive codes for distributed source coding," EURASIP Signal Processing, vol. 86, no. 11, pp. 3123-3130, November 2006. 